\title{
The number of spanning trees in a class of double fixed-step loop networks
}

\author{
Xuerong Yong*, Yuanping Zhang†, and Mordecai J. Golin ${ }^{\ddagger}$
}

\begin{abstract}
In this paper, we develop a method to count the number of spanning trees in certain classes of double fixed-step loop networks with nonconstant steps. More specifically our technique finds the number of spanning trees in $\vec{C}_{n}^{p, q}$, the double fixed-step loop network with $n$ vertices and jumps of size $p$ and $q$, when $n=d_{1} m$, and $q=d_{2} m+p$ where $d_{1}, d_{2}, p$ are arbitrary parameters and $m$ is a variable.
\end{abstract}

Key words: circulant digraph, spanning tree, Matrix Tree Theorem

\section{Introduction}

A directed circulant graph $\vec{C}_{n}^{s_{1}, s_{2}, \ldots, s_{k}}$ is a digraph on $n$ vertices $0,1,2, \ldots$, $n-1$; for each vertex $i(0 \leq i \leq n-1)$, there are $k$ arcs from $i$ to vertices $i+s_{1}, i+s_{2}, \ldots, i+s_{k}(\bmod n)$. (Figure 1 illustrates $\vec{C}_{6}^{4,5}$.) A double fixed-step loop network is a directed circulant graph $\vec{C}_{n}^{p, q}$ in which each vertex has exactly two arcs leaving it. This kind of network appears in the design and analysis of local area networks, multi-module memory organizations and parallel processing architectures $[1,6,9]$. Parameters of these graphs such as diameter and average distance, which are closely related to the network bandwidth, have been considered recently. For example, the case $\vec{C}_{n}^{1, n-1}$ and the case $\vec{C}_{n}^{1, n-2}$, the so called daisy-chain loop, were investigated by Liu [12]. Some generalizations to infinite classes of double fixed-step loop networks with minimum diameter

*Department of Mathematics, University of Puerto Rico at Mayaguez, PR 00681-9018, USA, xryong@math.uprm.edu.

${ }^{\dagger}$ School of Computer and Communication, Lanzhou University of Technology, Lanzhou, 730050, P. R. China, ypzhang@lut.cn.

${ }^{\ddagger}$ Department of Computer Science, The Hong Kong University of Science and Technology, Clear Water Bay, Kowloon, Hong Kong, golin@cs.ust.hk. 
were explored by Erdös and Hsu [5]. More recent results can be found in [1, 6, 9] and their references.

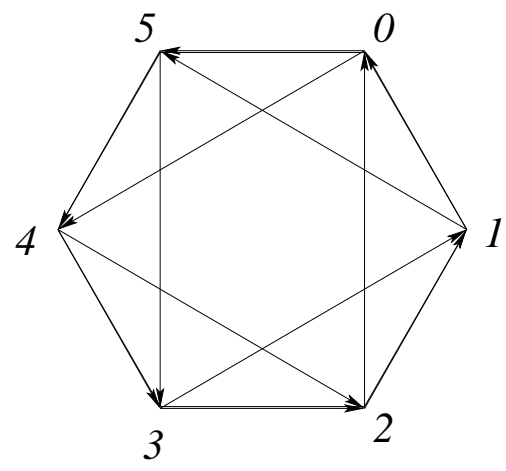

Figure 1: The directed circulant graph $\vec{C}_{6}^{4,5}$.

In this paper we address the question of counting the number of spanning trees in such digraphs. A spanning tree in a digraph is a rooted tree with directed paths from the root to all vertices. The number of spanning trees in a digraph or graph is an important, well studied quantity [4]. This parameter characterizes the reliability of networks. There is a classic result known as the Matrix Tree Theorem [11], which expresses the number of spanning trees $T(G)$ of a graph $G$ as a function of the determinant of a matrix that can be easily constructed from $G$ 's incidence matrix. However, in practice, counting the spanning trees by calculating the determinant is infeasible for large graphs. For this reason, researchers have paid much attention to developing techniques or deriving formulas for analyzing the number of spanning trees. For some special classes of graphs, it is possible to give explicit, simple formulae for the number of trees. For example, if $G$ is the complete graph $K_{n}$, then Cayley's tree formula [8] states that $T\left(K_{n}\right)=n^{n-2}$. Other special cases can be found in $[3,14,17]$.

The asymptotic behavior of $T\left(\vec{C}_{n}^{1, q}\right)$ has been derived in [15]. A closed formula for $T\left(\vec{C}_{n}^{1,2}\right)$ was proved in [13] where it was also proved that $T\left(\vec{C}_{n}^{1,2}\right) \geq$ $T\left(\vec{C}_{n}^{k, l}\right)$ for any different positive integers $k, l$.

For fixed integers $s_{1}, s_{2}, \ldots, s_{k}, 1 \leq s_{1}<s_{2}<\cdots<s_{k}$, it was proved in [14] and [17] that $T\left(\vec{C}_{n}^{s_{1}, s_{2}, \ldots, s_{k}}\right)=n a_{n}$, where $a_{n}$ satisfies a linear recurrence relation of order $2^{s_{k}-1}$. This recurrence relation can be exactly derived by using the Matrix Tree Theorem to calculate $a_{n}=T\left(\vec{C}_{n}^{s_{1}, s_{2}, \ldots, s_{k}}\right) / n$ for $n=$ $1,2,3, \ldots, 2^{s_{k}}$ which gives the initial conditions and enough information to solve 
for the coefficients of the recurrence relation.

The technique is not applicable, though, when the jumps $s_{i}$ vary with $n$. To the best of our knowledge, only a few very special cases of such graphs, e.g., the Möbius ladder [3], have been studied. Recently, Golin et al. [7] proved that, when the jumps are linear in the graph size, the number of spanning trees (as a function of the graph size) also satisfies a linear recurrence relation. Their proof was an existence one, though. Constructing the recurrence relation based on their existence proof would require calculating a very large number of initial values and is thus infeasible except for a few simple cases. In this paper, we will consider the number of spanning trees in a class of double fixed-step loop networks with jumps linear in the graph size. More specifically, we will develop a method for calculating $T\left(\vec{C}_{n}^{p, q}\right)$ when $n=d_{1} m$, and $q=d_{2} m+p$ where $d_{1}, d_{2}, p$ are arbitrary parameters and $m$ is a variable. In the next section we introduce our technique by developing all the necessary mathematical tools; in Section 3 we illustrate the technique by deriving the following series of formulas:

$$
\begin{aligned}
T\left(\vec{C}_{2 m}^{1, m+1}\right) & =m 2^{2 m-1}, \\
T\left(\vec{C}_{2 m}^{2, m+2}\right) & = \begin{cases}m 2^{2 m-1} & \text { if } 2 \nmid m, \\
0 & \text { otherwise, }\end{cases} \\
T\left(\vec{C}_{4 m}^{1, m+1}\right) & =m\left(2^{4 m-1}+2^{3 m-1}+2^{\frac{7 m}{2}} \cos \frac{m-2}{4} \pi\right), \\
T\left(\vec{C}_{3 m}^{1, m+1}\right) & =m\left(2^{3 m-1}+2^{m-1}-2^{2 m} \cos \frac{m-2}{3} \pi\right), \\
T\left(\vec{C}_{3 m}^{1,2 m+1}\right) & =m\left(2^{3 m-1}+2^{m-1}+2^{2 m} \cos \frac{m-1}{3} \pi\right), \\
T\left(\vec{C}_{3 m}^{2, m+2}\right) & = \begin{cases}m\left(2^{3 m-1}+2^{m-1}+2^{2 m} \cos \frac{m-1}{3} \pi\right) & \text { if } 2 \nmid m, \\
0 & \text { otherwise. }\end{cases} \\
T\left(\vec{C}_{3 m}^{2,2 m+2}\right) & = \begin{cases}m\left(2^{3 m-1}+2^{m-1}-2^{2 m} \cos \frac{m-2}{3} \pi\right) & \text { if } 2 \nmid m, \\
0 & \text { otherwise. }\end{cases} \\
T\left(\vec{C}_{3 m}^{3, m+3}\right) & =T\left(\vec{C}_{3 m}^{3,2 m+3}\right)= \begin{cases}m\left(2^{3 m-1}+2^{m-1}-2^{2 m} \cos \frac{m}{3} \pi\right) & \text { if } 3 \nmid m, \\
0 & \text { otherwise. }\end{cases}
\end{aligned}
$$

We point out that the basis of our method is not the fact that our digraphs are circulants but two consequences of the fact that they are circulants: (i) they are regular digraphs and (ii) the $j$ th eigenvalue of the digraph can be expressed as $f\left(\varepsilon^{j}\right), j=1,2, \ldots n$, where $f(x)$ is a polynomial and $\varepsilon=e^{\frac{2 \pi i}{n}}[2]$. Our technique could be extended to other graph classes with these properties as well. 
We also point out that our technique is restricted to counting the number of labeled spanning trees and can not count the number of non-isomorphic spanning trees. This is because the Matrix Tree Theorem counts the number of labeled spanning trees and our technique is an application of the Matrix Tree Theorem. An interesting open question would be to develop a method for counting the number of non-isomorphic spanning trees for these graph classes.

\section{The Basic Idea}

Our approach is based on the following result:

Lemma 1 (Zhang and Yong [16]) For any positive integers $n, p$ and $q$

$$
T\left(\vec{C}_{n}^{p, q}\right)=\prod_{j=1}^{n-1}\left(2-\varepsilon^{p j}-\varepsilon^{q j}\right)
$$

where $\varepsilon=e^{\frac{2 \pi i}{n}}$.

This lemma and similar ones are at the basis of most analyses of the number of spanning trees of circulant graphs. They come from combining the Matrix Tree Theorem with observations concerning the eigenvalues of the adjacency matrices of circulant graphs; see e.g., [17] for more details. While the lemma does provide a formula for $T\left(\vec{C}_{n}^{p, q}\right)$ it is not a particularly useful one. The rest of this paper will be devoted to transforming (1) into something more interesting.

Lemma 2 Let $n, p$ and $q$ be any positive integers and $\varepsilon=e^{\frac{2 \pi i}{n}}$. Define $\delta_{1}, \delta_{2}$, $\ldots, \delta_{n}$ so that

$$
f(x)=\prod_{j=1}^{n}\left(x-\varepsilon^{p j}-\varepsilon^{j q}\right)=x^{n}-\delta_{1} x^{n-1}+\delta_{2} x^{n-2}-\cdots+(-1)^{n} \delta_{n} .
$$

Then

$$
T\left(\vec{C}_{n}^{p, q}\right)=f^{\prime}(2)=n 2^{n-1}+\sum_{j=1}^{n-1}(-1)^{j} \delta_{j}(n-j) 2^{n-j-1} .
$$

Proof. Note that $f(2)=0$, and from Lemma 1 we know

$$
T\left(\vec{C}_{n}^{p, q}\right)=\prod_{j=1}^{n-1}\left(2-\varepsilon^{p j}-\varepsilon^{q j}\right)
$$




$$
\begin{aligned}
& =\lim _{x \rightarrow 2} \prod_{j=1}^{n-1}\left(x-\varepsilon^{p j}-\varepsilon^{q j}\right) \\
& =\lim _{x \rightarrow 2} \frac{f(x)}{x-2}=\lim _{x \rightarrow 2} \frac{f(x)-f(2)}{x-2}=f^{\prime}(2) .
\end{aligned}
$$

To find $T\left(\vec{C}_{n}^{p, q}\right)$ our approach will therefore be to find $\delta_{1}, \delta_{2}, \ldots, \delta_{n-1}$ and substitute them into (2). Our main tool in calculating the $\delta_{i}$ will be Newton's formulae [10] which states the following:

- Let $x_{1}, x_{2}, \ldots, x_{n}$ be arbitrary values.

- Let $\delta_{1}, \delta_{2}, \ldots, \delta_{n}$ be the coefficients of

$$
f(x)=\left(x-x_{1}\right)\left(x-x_{2}\right) \cdots\left(x-x_{n}\right)=x^{n}-\delta_{1} x^{n-1}+\cdots+(-1)^{n} \delta_{n} .
$$

- For $i=1,2, \ldots, n$, define $\mathcal{S}_{i}=x_{1}^{i}+x_{2}^{i}+\cdots+x_{n}^{i}$.

- Then Newton's formulae are, for $i=1,2, \ldots, n$ :

$$
\mathcal{S}_{i}-\delta_{1} \mathcal{S}_{i-1}+\delta_{2} \mathcal{S}_{i-2}+\cdots+(-1)^{i-1} \delta_{i-1} \mathcal{S}_{1}+(-1)^{i} i \delta_{i}=0
$$

More specifically, Newton's formulae permit us to derive the $\delta_{i}$ through knowledge of the $\mathcal{S}_{i}$. Note that the roots of $f(x)$ in Lemma 2 are $\left(\varepsilon^{k p}+\varepsilon^{k q}\right), k=$ $1,2, \ldots, n$. In the following lemma and the sequel we use $C_{n}^{j}$ to denote $\left(\begin{array}{l}n \\ j\end{array}\right)$.

Lemma 3 Let

$$
S_{i}=\sum_{k=1}^{n}\left(\varepsilon^{k p}+\varepsilon^{k q}\right)^{i}, i=1,2, \ldots, n,
$$

where $\varepsilon=e^{\frac{2 \pi i}{n}}$. Then, for $1 \leq i \leq n$ :

$$
S_{i}=n \sum_{\substack{0 \leq j \leq i \\ p i+(q-p) j=0}} C_{i}^{j} .
$$

Proof. For $1 \leq i \leq n$

$$
\begin{aligned}
S_{i} & =\sum_{k=1}^{n}\left(\varepsilon^{k p}+\varepsilon^{k q}\right)^{i} \\
& =\sum_{k=1}^{n} \sum_{j=0}^{i} C_{i}^{j} \varepsilon^{k p i+k(q-p) j}
\end{aligned}
$$




$$
\begin{aligned}
& =\sum_{j=0}^{i} C_{i}^{j}\left(1+\varepsilon^{p i+(q-p) j}+\varepsilon^{2(p i+(q-p) j)}+\cdots+\varepsilon^{(n-1)(p i+(q-p) j)}\right) \\
& =n \sum_{\substack{0 \leq j \leq i \\
p i+(q-p) j=0(\bmod n)}} C_{i}^{j} .
\end{aligned}
$$

In the most general case of arbitrary $n, p, q$ this lemma does not help us much since the sums involved are quite complicated. However, in the particular cases in which $q=d_{2} m+p, n=d_{1} m$ where $p$ and $d_{2}<d_{1}$ are fixed and $m$ grows, we can greatly simplify this sum, as shown in the next corollary:

Corollary 1 Let $p, d_{1}$ and $d_{2}$ with $d_{2}<d_{1}$ be fixed, $n=d_{1} m$, and $q=d_{2} m+p$. Set

$$
S_{i}=\sum_{k=1}^{n}\left(\varepsilon^{k p}+\varepsilon^{k q}\right)^{i}, i=1,2, \ldots, n,
$$

where $\varepsilon=e^{\frac{2 \pi i}{n}}$. Further define

$$
\begin{aligned}
\alpha & =\operatorname{gcd}(p, m), \quad \quad p^{\prime}=p / \alpha \\
\beta & =\operatorname{gcd}\left(d_{1}, d_{2}\right), \\
\gamma & =\operatorname{gcd}\left(\beta, p^{\prime}\right), \\
d_{1}^{\prime} & =d_{1} / \beta, \\
d_{2}^{\prime} & =d_{2} / \beta
\end{aligned}
$$

and let $\bar{d}_{2}^{\prime}$ be such that $d_{2}^{\prime} \bar{d}_{2}^{\prime} \equiv 1 \quad\left(\bmod d_{1}^{\prime}\right)$. Then

$$
S_{i}= \begin{cases}0 & \text { if } \frac{\beta}{\gamma \alpha} m \nmid i, \\ n \sum_{t=0}^{\left\lfloor(i-x) / d_{1}^{\prime}\right\rfloor} C_{i}^{x+t d_{1}^{\prime}} & \text { if } i=\ell \frac{\beta}{\gamma \alpha} m, \ell=1,2, \ldots, \frac{d_{1} \alpha \gamma}{\beta},\end{cases}
$$

where $x=\left(-\ell p^{\prime \prime} \bar{d}_{2}^{\prime}\right) \bmod d_{1}^{\prime}$.

Proof. Recall from Lemma 3 that $S_{i}=n \sum_{\substack { p i+(q-p) j=0(\bmod n) \\ \begin{subarray}{c}{0 \leq j \leq i \\ j{ p i + ( q - p ) j = 0 ( \operatorname { m o d } n ) \\ \begin{subarray} { c } { 0 \leq j \leq i \\ j } }\end{subarray}} C_{i}^{j}$. In what follows we examine, for fixed $i$, which $j$ satisfy the condition

$$
p i+(q-p) j \equiv 0 \quad(\bmod n) .
$$

Relation (5) is equivalent to

$$
p i+d_{2} m j \equiv 0 \quad\left(\bmod d_{1} m\right)
$$


which can only be satisfied if $m \mid p i$ or, since $\alpha=\operatorname{gcd}(m, p)$, if $\frac{m}{\alpha} \mid i$. We may therefore assume that $i=\ell \frac{m}{\alpha}, \ell=1,2, \ldots, \alpha d_{1}$. Then

$$
\begin{aligned}
(5) \text { is satisfied } & \Longleftrightarrow \ell \frac{m}{\alpha} p+d_{2} m j \equiv 0 \quad\left(\bmod m d_{1}\right) \\
& \Longleftrightarrow \ell p^{\prime}+d_{2} j \equiv 0 \quad\left(\bmod d_{1}\right) \\
& \Longleftrightarrow d_{2} j \equiv\left(-\ell p^{\prime}\right) \quad\left(\bmod d_{1}\right) .
\end{aligned}
$$

Since $\beta=\operatorname{gcd}\left(d_{1}, d_{2}\right)$, if $\beta \nmid \ell p^{\prime}$ this last condition can not be satisfied so, if $S_{i} \neq 0$, then $\beta \mid \ell p^{\prime}$ or $\frac{\beta}{\gamma} \mid \ell \frac{p^{\prime}}{\gamma}$. Since $\gamma=\operatorname{gcd}\left(\beta, p^{\prime}\right)$, we may assume that $\ell=\frac{\beta}{\gamma} \ell^{\prime}$ for some integer $\ell^{\prime}$. This in turn implies that

$$
i=\ell \frac{m}{\alpha}=\ell^{\prime} \frac{\beta}{\gamma} \frac{m}{\alpha}
$$

and

(5) is satisfied $\Longleftrightarrow d_{2} j \equiv\left(-\ell p^{\prime}\right) \quad\left(\bmod d_{1}\right)$

$$
\begin{aligned}
& \Longleftrightarrow d_{2} j \equiv\left(-\ell^{\prime} \beta \frac{p^{\prime}}{\gamma}\right) \quad\left(\bmod d_{1}\right) \\
& \Longleftrightarrow d_{2}^{\prime} j \equiv\left(-\ell^{\prime} p^{\prime \prime}\right) \quad\left(\bmod d_{1}^{\prime}\right) \\
& \Longleftrightarrow j \equiv\left(-\ell^{\prime} p^{\prime \prime}\right) \bar{d}_{2}^{\prime} \quad\left(\bmod d_{1}^{\prime}\right)
\end{aligned}
$$

from which (4) follows.

We now note that even though we proved the corollary in full generality, in our spanning tree application we will not need this full generality. More specifically, we have the following result:

Lemma 4 If $\operatorname{gcd}(p, q, n)>1$ then

$$
T\left(\vec{C}_{n}^{p, q}\right)=0 .
$$

In particular, given $p, d_{1}, d_{2}$, let $\alpha=\operatorname{gcd}(p, m)$ and $\delta=\operatorname{gcd}\left(d_{1}, d_{2}, p\right)$. Then, if either $\alpha>1$ or $\delta>1$,

$$
T\left(\vec{C}_{d_{1} m}^{p, d_{2} m+p}\right)=0
$$

Proof. To prove (6) note that if $\operatorname{gcd}(n, p, q)>1$ and $(u, v)$ is an arc in $\vec{C}_{n}^{p, q}$ then $u \equiv v \quad(\bmod \operatorname{gcd}(n, p, q))$. This implies that if $u^{\prime}, v^{\prime}$ are any two vertices in $\vec{C}_{n}^{p, q}$ and there is a path from $u^{\prime}$ to $v^{\prime}$ then $u^{\prime} \equiv v^{\prime} \quad(\bmod \operatorname{gcd}(n, p, q))$. This in turn implies that there is no one vertex in $\vec{C}_{n}^{p, q}$ from which it is possible to reach all of the vertices so $\vec{C}_{n}^{p, q}$ does not contain any spanning tree. 
(An alternative proof would be to note that, setting $j=\frac{n}{\operatorname{gcd}(n, p, q)}$ would give $\varepsilon^{j p}=\varepsilon^{j q}=1$ so $\left(2-\varepsilon^{j p}-\varepsilon^{j q}\right)=0$ and, from $(1), T\left(\vec{C}_{n}^{p, q}\right)=\prod_{j=1}^{n-1}(2-$ $\left.\varepsilon^{p j}-\varepsilon^{q j}\right)=0$.)

To prove the second part of the lemma simply note that if either $\alpha>1$ or $\delta>1$ then $\operatorname{gcd}\left(p, d_{2} m+p, d_{1} m\right)>1$.

In the sequel we may therefore assume that (i) $\alpha=\operatorname{gcd}(p, m)=1$ so $p^{\prime}=$ $p / \alpha=p$ and therefore (ii) $\gamma=\operatorname{gcd}\left(\beta, p^{\prime}\right)=\operatorname{gcd}\left(d_{1}, d_{2}, p\right)=1$ as well. This then implies $p^{\prime \prime}=p^{\prime} / \gamma=p \gamma=p$. We will use this fact later in Section 3 .

Returning to the corollary we observe from (4) that all of the $S_{i}$ are 0 except for those that are some multiple of $\frac{\beta}{\alpha} m=\beta m$. We make a further observation.

Lemma 5 Given $x_{1}, x_{2}, \ldots, x_{n}$, let $\delta_{1}, \delta_{2}, \ldots, \delta_{n}$ be defined by

$$
f(x)=\left(x-x_{1}\right)\left(x-x_{2}\right) \cdots\left(x-x_{n}\right)=x^{n}-\delta_{1} x^{n-1}+\cdots+(-1)^{n} \delta_{n} .
$$

For $i=1,2, \ldots, n$, set $\mathcal{S}_{i}=x_{1}^{i}+x_{2}^{i}+\cdots+x_{n}^{i}$. If there exists an integer $v$ such that

$$
\forall i, 1 \leq i \leq n, \text { if } v \nmid i \text { then } S_{i}=0,
$$

then

$$
\forall i, 1 \leq i \leq n \text {, if } v \nmid i \text { then } \delta_{i}=0 \text {. }
$$

Proof. We assume that $v>1$ since otherwise the lemma is trivially correct. Now assume that if $v \nmid i$ then $S_{i}=0$. We prove by induction on $i$ that if $v \nmid i$ then $\delta_{i}=0$.

Note that we can rewrite Newton's formulae (3) as

$$
\delta_{i}=\frac{(-1)^{i+1}}{i}\left(S_{i}+\sum_{t=1}^{i-1}(-1)^{t} \delta_{t} S_{i-t}\right) .
$$

For $i=1, \delta_{1}=S_{1}=0$. Suppose now that $\delta_{j}=0$ for all $j<i$ such that $v \nmid j$. If $v \nmid i$ then, $\forall t<i$, at least one of $v \nmid t$ or $v \nmid(i-t)$ is true so $\delta_{t} S_{i-t}=0$ and, since $S_{i}=0$ we have from (7) that $\delta_{i}=0$.

Now (recalling from the comment following Lemma 4 that $\alpha=1$ ) set $v=$ $\frac{\beta}{\gamma} m=\beta m$. From Corollary 1 we know that if $v \nmid i$ then $S_{i}=0$. Lemma 5 then implies that if $v \nmid i$ then $\delta_{i}=0$. To solve for $\delta_{i}$ when $v \mid i$ we can rewrite Newton's formulae, simplifying by discarding all zero terms to get

$$
\begin{aligned}
& 0=S_{v}+(-1)^{v} v \delta_{v} \\
& 0=S_{2 v}+(-1)^{v} \delta_{v} S_{v}+(-1)^{2 v} 2 v \delta_{2 v}
\end{aligned}
$$




$$
\begin{aligned}
0 & =S_{3 v}+(-1)^{v} \delta_{v} S_{2 v}+(-1)^{2 v} \delta_{2 v} S_{v}+(-1)^{3 v} 3 v \delta_{3 v} \\
\vdots & \vdots \\
0 & =
\end{aligned}
$$

where $d=\frac{d_{1} \alpha}{\beta}=\frac{d_{1}}{\beta}=d_{1}^{\prime}$. Now for $i=1,2, \ldots, d$ set $Y_{i}(v)=S_{i v}$ to be the known functions and $X_{i}(v)=\delta_{i v}$ to be the functions for which we want to solve. The system above then becomes

$$
\begin{aligned}
-Y_{1}(v) & =(-1)^{v} v X_{1}(v) \\
-Y_{2}(v) & =(-1)^{v} X_{1}(v) Y_{1}(v)+(-1)^{2 v} 2 v X_{2}(v) \\
-Y_{3}(v) & =(-1)^{v} X_{1}(v) Y_{2}(v)+(-1)^{2 v} X_{2}(v) Y_{1}(v)+(-1)^{3 v} 3 v X_{3}(v) \\
\vdots & \vdots \\
-Y_{(d-1)}(v) & =(-1)^{v} X_{1}(v) Y_{(d-2)}(v)+\cdots+(-1)^{(d-1) v}(d-1) v X_{(d-1)}(v) \\
-Y_{d}(v) & =(-1)^{v} X_{1}(v) Y_{(d-1)}(v)+\cdots+(-1)^{(d-1) v} X_{(d-1) v} Y_{1}(v)+(-1)^{d v} d v X_{d}(v)
\end{aligned}
$$

which is non-singular and can therefore be solved for $X_{i}(v)$ in terms of the $Y_{i}(v)$. In the next section we see examples of this technique.

As pointed out in the comments after Lemma 2 we do not need to know $\delta_{n}=X_{d}(v)$ to calculate $T\left(\vec{C}_{n}^{p, q}\right)$ so we actually only need to solve for the $d-1$ functions $X_{i}(v)=\delta_{i v}, i=1,2, \ldots, d-1$ and not all the $d$ functions.

Before proceeding to calculate $T\left(\vec{C}_{n}^{p, q}\right)$ we note that the expression for $Y_{i}(v)=S_{i v}$ given in equation (4) of Corollary 1 is in the form of a sum of binomial coefficients of an arithmetic series. While this looks unwieldy we will actually be able to use the following useful lemma to derive a closed form for the sums.

Lemma 6 Let $n \geq 0$ and let $j, d$ satisfy $0 \leq j \leq d-1$. Then

$$
C_{n}^{j}+C_{n}^{d+j}+C_{n}^{2 d+j}+\cdots+C_{n}^{\left\lfloor\frac{n-j}{d}\right\rfloor d+j}=\frac{2^{n}}{d} \sum_{k=0}^{d-1} \cos ^{n} \frac{k}{d} \pi \cos \frac{k(n-2 j)}{d} \pi .
$$

Proof. Let $\omega=e^{\frac{2 \pi i}{d}}$. So $\omega$ is the $d$ th root of unity and

$$
\sum_{j=0}^{d-1}\left(\omega^{k}\right)^{j}= \begin{cases}d & k=0(\bmod d) \\ 0 & \text { otherwise }\end{cases}
$$

For each $k, 0 \leq k \leq d-1$, multiply both sides of the following identity by $\omega^{k(d-j)}$ :

$$
\sum_{l=0}^{n} C_{n}^{l}\left(\omega^{k}\right)^{l}=\left(1+\omega^{k}\right)^{n}
$$


Summing up the $d$ identities for $k=0,1, \ldots, d-1$ yields

$$
d\left(C_{n}^{j}+C_{n}^{d+j}+C_{n}^{2 d+j}+\cdots+C_{n}^{\left\lfloor\frac{n-j}{d}\right\rfloor d+j}\right)=\sum_{k=0}^{d-1} \omega^{k(d-j)}\left(1+\omega^{k}\right)^{n} .
$$

Since

$$
\begin{aligned}
\sum_{k=0}^{d-1} \omega^{k(d-j)}\left(1+\omega^{k}\right)^{n} & =\sum_{k=0}^{d-1} e^{\frac{2 k(d-j) \pi i}{d}}\left(1+e^{\frac{2 k \pi i}{d}}\right)^{n} \\
& =\sum_{k=0}^{d-1} e^{\frac{2 k(d-j) \pi i}{d}}\left(2 \cos \frac{k}{d} \pi e^{\frac{k \pi i}{d}}\right)^{n} \\
& =2^{n} \sum_{k=0}^{d-1} \cos ^{n} \frac{k}{d} \pi e^{\frac{k(n-2 j) \pi i}{d}}
\end{aligned}
$$

taking the real part of (9) proves the lemma.

\section{The Technique and Examples}

In this section we use the facts developed in the previous section to derive formulas for $T\left(\vec{C}_{n}^{p, q}\right)$ as a function of $m$ when $n=d_{1} m$ and $q=d_{2} m+p$. Recall that from Lemma 4, we may assume that both $\alpha=\operatorname{gcd}(p, m)=1$ and $\gamma=\operatorname{gcd}\left(d_{1}, d_{2}, p\right)=1$ since, if not, $T\left(\vec{C}_{d_{1} m}^{p, d_{2} m+p}\right)=0$. Furthermore, from the comments following the lemma, we may also assume that $p^{\prime \prime}=p^{\prime} / \gamma=$ $(p / \alpha) / \gamma=p$.

\subsection{The Technique}

Gathering together all of the facts from the previous section yields the following four step technique.

1. Calculate $\beta=\operatorname{gcd}\left(d_{1}, d_{2}\right), d_{1}^{\prime}=d_{1} / \beta, d_{2}^{\prime}=d_{2} / \beta$ and $\overline{d_{2}^{\prime}}$ such that $d_{2}^{\prime} \bar{d}_{2}^{\prime} \equiv$ $1\left(\bmod d_{1}^{\prime}\right)$.

2. Set $v=m \beta$. For $\ell=1,2, \ldots, d_{1}^{\prime}-1$ use Corollary 1 and Lemma 6 to calculate

$$
\begin{aligned}
Y_{\ell}(v) & =S_{\ell v} \\
& =n \sum_{t=0}^{\left\lfloor\left(\ell v-x_{\ell}\right) / d_{1}^{\prime}\right\rfloor} C_{\ell v}^{x_{\ell}+t d_{1}^{\prime}}
\end{aligned}
$$




$$
\begin{aligned}
& =n \frac{2^{\ell v}}{d_{1}^{\prime}} \sum_{k=0}^{d_{1}^{\prime}-1}\left(\cos ^{\ell} \frac{k}{d_{1}^{\prime}} \pi\right)^{v} \cos \frac{k\left(\ell v-2 x_{\ell}\right)}{d_{1}^{\prime}} \pi \\
& =v 2^{\ell v} \sum_{k=0}^{d_{1}^{\prime}-1}\left(\cos \frac{k}{d_{1}^{\prime}} \pi\right)^{\ell v} \cos \frac{k\left(\ell v-2 x_{\ell}\right)}{d_{1}^{\prime}} \pi
\end{aligned}
$$

where $x_{\ell}=\left(-\ell p^{\prime \prime} \bar{d}_{2}^{\prime}\right) \bmod d_{1}^{\prime}=\left(-\ell p \overline{d_{2}^{\prime}}\right) \bmod d_{1}^{\prime}$.

3. Use (8) to solve for $\delta_{\ell v}=X_{\ell}(v), \ell=1,2, \ldots, d_{1}^{\prime}-1$.

4. Substitute the derived $\delta_{\ell v}$ values into (2) and use the fact that if $v \nmid i$ then $\delta_{i}=0$ to derive

$$
T\left(\vec{C}_{n}^{p, q}\right)=f^{\prime}(2)=n 2^{n-1}+\sum_{\ell=1}^{d_{1}^{\prime}-1}(-1)^{\ell \beta m}(n-\ell \beta m) \delta_{\ell \beta m} 2^{n-\ell \beta m-1} .
$$

We also make two observations that can reduce the number of cases that need to be examined. The first is simply that if $\beta=\operatorname{gcd}\left(d_{1}, d_{2}\right), d_{1}^{\prime}=d_{1} / \beta$ and $d_{2}^{\prime}=d_{2} / \beta$, then setting $g(m)=T\left(\vec{C}_{d_{1}^{\prime} m}^{p, d_{2}^{\prime} m+p}\right)$ and $h(m)=T\left(\vec{C}_{d_{1} m}^{p, d_{2} m+p}\right)$ gives $h(m)=g(\beta m)$. Since, in our technique, solving for both $g(m)$ and $h(m)$ involve the 'same amount of work', i.e., solving for $d^{\prime}-1$ unknowns from $d^{\prime}-1$ equations, we might as well solve for $g(m)$. For example, instead of solving for $T\left(\vec{C}_{6 m}^{1,4 m+1}\right)$ we can solve for $T\left(\vec{C}_{3 m}^{1,2 m+1}\right)$.

The second more interesting observation is stated next.

Lemma 7 Let $\beta=\operatorname{gcd}\left(d_{1}, d_{2}\right), d_{1}^{\prime}=d_{1} / \beta$ and $d_{2}^{\prime}=d_{2} / \beta$. If $p_{1} \equiv p_{2} \quad\left(\bmod d_{1}^{\prime}\right)$, $\operatorname{gcd}\left(p_{1}, m\right)=\operatorname{gcd}\left(p_{2}, m\right)=1$, and $\operatorname{gcd}\left(\beta, p_{1}\right)=\operatorname{gcd}\left(\beta, p_{2}\right)=1$, then

$$
T\left(\vec{C}_{d_{1} m}^{p_{1}, d_{2} m+p_{1}}\right)=T\left(\vec{C}_{d_{1} m}^{p_{2}, d_{2} m+p_{2}}\right) .
$$

Proof. Examining our technique for deriving $T\left(\vec{C}_{d_{1} m}^{p, d_{2} m+p}\right)$ we note that the only place in which $p$ is used is in the definition of $x_{\ell}=\left(-\ell p \overline{d_{2}^{\prime}}\right) \quad\left(\bmod d_{1}^{\prime}\right)$ in Step 2. This value is the same for all $\ell$ if $p_{1} \equiv p_{2} \quad\left(\bmod d_{1}^{\prime}\right)$ so the proof follows. The reason for the requirement that $\operatorname{gcd}\left(p_{1}, m\right)=\operatorname{gcd}\left(p_{2}, m\right)=1$ is that in Step 2 we were explicitly using the fact that $\operatorname{gcd}(p, m)=1$ to force $p^{\prime \prime}=p$. As seen before, if $\operatorname{gcd}(p, m) \neq 1$ then the graph has no spanning trees so this is not an interesting case.

As an example this lemma would imply that

$$
\begin{aligned}
& \text { if } 2 \nmid m \text { then } \\
& \text { if } 2 \nmid m \text { and } 5 \nmid m \text { then } \\
& \text { for all } m
\end{aligned}
$$

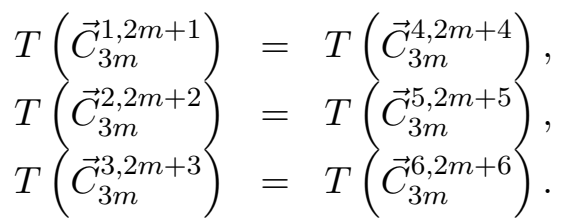


Note that if $2 \mid m$ then $T\left(\vec{C}_{3 m}^{4,2 m+4}\right)=0, T\left(\vec{C}_{3 m}^{2,2 m+2}\right)=0$ and $T\left(\vec{C}_{3 m}^{6,2 m+6}\right)=$ 0 ; if $5 \mid m$ then $T\left(\vec{C}_{3 m}^{5,2 m+5}\right)=0$; while if $3 \mid m$ then $T\left(\vec{C}_{3 m}^{3,2 m+3}\right)=T\left(\vec{C}_{3 m}^{6,2 m+6}\right)=$ 0.

\subsection{Examples}

We illustrate this technique by first evaluating the simplest case.

Example 1: $T\left(\vec{C}_{2 m}^{1, m+1}\right)$.

In this case $p=d_{2}=1, d_{1}=2$ so $\beta=1, v=m$ and $d_{1}^{\prime}=d_{1} / \beta=2$. Since $d_{1}^{\prime}=2$ we only need to find $\delta_{v}=X_{1}(v)$. Note that $\bar{d}_{2}^{\prime}=d_{2}^{\prime}=1$ so $x_{1}=(-1) \bmod 2=1$. Substituting into (10) gives

$$
Y_{1}(v)=S_{m}=2 m \sum_{t=0}^{\left\lfloor\frac{m-1}{2}\right\rfloor} C_{m}^{2 t+1}=2 m 2^{m-1} .
$$

The system of equations (8) in this case is only the one equation

$$
-Y_{1}(v)=(-1)^{v} v X_{1}(v)
$$

or

$$
-2 m 2^{m-1}=-Y_{1}(v)=(-1)^{v} v X_{1}(v)=(-1)^{m} m \delta_{m},
$$

so

$$
\delta_{m}=(-1)^{m+1} 2^{m} .
$$

Substituting into (11) yields

$$
T\left(\vec{C}_{2 m}^{1, m+1}\right)=(2 m) 2^{2 m-1}+(-1)^{m} m \delta_{m} 2^{m-1}=m 2^{2 m-1} .
$$

Example 2: $T\left(\vec{C}_{2 m}^{2, m+2}\right)$

In this case $p=d_{1}=2, d_{2}=1, \beta=1$ so $v=m, d_{1}^{\prime}=d_{1} / \beta=2$.

The major difference in this case is that we must note that if $\alpha=$ $\operatorname{gcd}(m, p) \neq 1$, i.e., $m$ is even, then $T\left(\vec{C}_{2 m}^{2, m+2}\right)=0$. So, for the rest of the derivation we assume that $m$ is odd. 
Since $d_{1}^{\prime}=2$ we only need to find $\delta_{v}=X_{1}(v)$. Note that $\bar{d}_{2}^{\prime}=d_{2}^{\prime}=1$ so $x_{1}=(-1 \cdot 2) \bmod 2=0$. Substituting into (10) gives

$$
Y_{1}(v)=S_{m}=2 m \sum_{t=0}^{\left\lfloor\frac{m}{2}\right\rfloor} C_{m}^{2 t}=2 m 2^{m-1} .
$$

$Y_{1}(v)=2 m 2^{m-1}$ is exactly the same as (12) so following the same derivation as in (12)-(13) we find, that if $m$ is odd then

$$
T\left(\vec{C}_{2 m}^{2, m+2}\right)=(2 m) 2^{2 m-1}+(-1)^{m} m \delta_{m} 2^{m-1}=m 2^{2 m-1} .
$$

Thus

$$
T\left(\vec{C}_{2 m}^{2, m+2}\right)= \begin{cases}m 2^{2 m-1} & \text { if } m \text { is odd } \\ 0 & \text { if } m \text { is even }\end{cases}
$$

Example 3: $T\left(\vec{C}_{4 m}^{1, m+1}\right)$.

In this case $p=d_{2}=1, d_{1}=4, \beta=1$ so $v=m, d_{1}^{\prime}=d_{1} / \beta=4$. We therefore only need to derive the three functions $\delta_{\ell v}=X_{\ell}(v), \ell=1,2,3$. As before $\bar{d}_{2}^{\prime}=d_{2}^{\prime}=1$. We therefore find $x_{1}=3, \quad x_{2}=2, \quad x_{3}=1$. Substituting into (10) yields

$$
\begin{aligned}
& Y_{1}(v)=v\left(2^{v}+2^{\frac{v}{2}+1} \cos \frac{v-6}{4} \pi\right) \\
& Y_{2}(v)=v\left(2^{2 v}+2^{v+1} \cos \frac{v-2}{2} \pi\right), \\
& Y_{3}(v)=v\left(2^{3 v}+2^{\frac{3 v}{2}+1} \cos \frac{3 v-2}{4} \pi\right) .
\end{aligned}
$$

The system of equations (8) in this case is

$$
\begin{aligned}
& -Y_{1}(v)=(-1)^{v} v X_{1}(v) \\
& -Y_{2}(v)=(-1)^{v} X_{1}(v) Y_{1}(v)+(-1)^{2 v} 2 v X_{2}(v) \\
& -Y_{3}(v)=(-1)^{v} X_{1}(v) Y_{2}(v)+(-1)^{2 v} X_{2}(v) Y_{1}(v)+(-1)^{3 v} 3 v X_{3}(v),
\end{aligned}
$$

and solving for $X_{\ell}(v)$ yields

$$
\begin{aligned}
& X_{1}(v)=(-1)^{v}\left(-2^{v}+2^{\frac{v}{2}+1} \cos \frac{v-2}{4} \pi\right), \\
& X_{2}(v)=(-1)^{2 v}\left(2^{v}-2^{\frac{3 v}{2}+1} \cos \frac{v-2}{4} \pi\right), \\
& X_{3}(v)=(-1)^{3 v}\left(-2^{2 v}\right) .
\end{aligned}
$$


Equation (11) states

$T\left(\vec{C}_{4 m}^{1, m+1}\right)=4 m 2^{4 m-1}+(-1)^{m} 3 m \delta_{m} 2^{3 m-1}+(-1)^{2 m} 2 m \delta_{2 m} 2^{2 m-1}+(-1)^{3 m} m \delta_{3 m} 2^{m-1}$.

Substituting in the values $\delta_{\ell m}=X_{\ell}(m)$ and simplifying yields

$$
T\left(\vec{C}_{4 m}^{1, m+1}\right)=m\left(2^{4 m-1}+2^{3 m-1}+2^{\frac{7 m}{2}} \cos \frac{m-2}{4} \pi\right) .
$$

Example 4: $T\left(\vec{C}_{3 m}^{p, d_{2} m+p}\right)$ where $1 \leq p \leq 3,1 \leq d_{2} \leq 2$.

We will first work through the six different $(p, d)$ cases, stating our results and showing that there are actually only three distinct cases (with the remaining three being isomorphic to the others except, possibly, when they are equal to 0 ). We will then derive the formulas for the three distinct cases at the end of the section.

(i) $T\left(\vec{C}_{3 m}^{1, m+1}\right)$.

$$
T\left(\vec{C}_{3 m}^{1, m+1}\right)=m\left(2^{3 m-1}+2^{m-1}-2^{2 m} \cos \frac{m-2}{3} \pi\right) .
$$

(ii) $T\left(\vec{C}_{3 m}^{1,2 m+1}\right)$.

$$
T\left(\vec{C}_{3 m}^{1,2 m+1}\right)=m\left(2^{3 m-1}+2^{m-1}+2^{2 m} \cos \frac{m-1}{3} \pi\right) .
$$

(iii) $T\left(\vec{C}_{3 m}^{3,2 m+3}\right)$.

$$
T\left(\vec{C}_{3 m}^{3,2 m+3}\right)= \begin{cases}m\left(2^{3 m-1}+2^{m-1}-2^{2 m} \cos \frac{m}{3} \pi\right) & \text { if } 3 \nmid m, \\ 0 & \text { otherwise. }\end{cases}
$$

(iv) $T\left(\vec{C}_{3 m}^{2, m+2}\right)$.

When $m$ is even $T\left(\vec{C}_{3 m}^{2, m+2}\right)=0$.

When $m$ is odd $\vec{C}_{3 m}^{2, m+2}$ is isomorphic to $\vec{C}_{3 m}^{1,2 m+1}$. More explicitly,

$$
\vec{C}_{3 m}^{1,2 m+1} \Longleftrightarrow \vec{C}_{3 m}^{2, m+2}
$$

with the trivial vertex mapping $i \longleftrightarrow 2 i$. Therefore

$$
T\left(\vec{C}_{3 m}^{2, m+2}\right)= \begin{cases}m\left(2^{3 m-1}+2^{m-1}+2^{2 m} \cos \frac{m-1}{3} \pi\right) & \text { if } 2 \nmid m, \\ 0 & \text { otherwise }\end{cases}
$$


(v) $T\left(\vec{C}_{3 m}^{2,2 m+2}\right)$.

When $m$ is even $T\left(\vec{C}_{3 m}^{2,2 m+2}\right)=0$.

When $m$ is odd $\vec{C}_{3 m}^{2,2 m+2}$ is isomorphic to $\vec{C}_{3 m}^{1, m+1}$ using the mapping

$$
\vec{C}_{3 m}^{1, m+1} \Longleftrightarrow \vec{C}_{3 m}^{2,2 m+2}
$$

with the same trivial vertex mapping $i \longleftrightarrow 2 i$.

Therefore

$$
T\left(\vec{C}_{3 m}^{2,2 m+2}\right)= \begin{cases}m\left(2^{3 m-1}+2^{m-1}-2^{2 m} \cos \frac{m-2}{3} \pi\right) & \text { if } 2 \nmid m, \\ 0 & \text { otherwise. }\end{cases}
$$

(vi) $T\left(\vec{C}_{3 m}^{3, m+3}\right)$.

If $3 \mid m$ then $T\left(\vec{C}_{3 m}^{3, m+3}\right)=0$. If $3 \nmid m$ then $\vec{C}_{3 m}^{3, m+3}$ is isomorphic to $\vec{C}_{3 m}^{3,2 m+3}$. To see this note that if $3 \nmid m$ then $\operatorname{gcd}(3 m, m+3)=1$ so there exists $x<3 m$ such that $x(m+3) \equiv 1 \quad(\bmod 3 m)$. We can then define the isomorphism

$$
\vec{C}_{3 m}^{3, m+3} \Longleftrightarrow \vec{C}_{3 m}^{3,2 m+3}
$$

using the vertex mapping $i \longleftrightarrow i x(2 m+3)$. To see this is an isomorphism let

$$
\begin{aligned}
f(i) & =i x(2 m+3) \quad(\bmod 3 m) \\
& =i x(m+3+m) \quad(\bmod 3 m) \\
& =i(1+x m) \quad(\bmod 3 m) .
\end{aligned}
$$

Then

$$
\begin{aligned}
f(i+3) & =(i+3)(1+x m) \quad(\bmod 3 m) \\
& =f(i)+3 \quad(\bmod 3 m)
\end{aligned}
$$

and

$$
\begin{aligned}
f(i+m+3) & =(i+m+3) x(2 m+3) \quad(\bmod 3 m) \\
& =i x(2 m+3)+(m+3) x(2 m+3) \quad(\bmod 3 m) \\
& =f(i)+2 m+3 \quad(\bmod 3 m) .
\end{aligned}
$$

So, if $(i, i+3) \in \vec{C}_{3 m}^{3, m+3}$ then $(f(i), f(i+3)) \in \vec{C}_{3 m}^{3,2 m+3}$ and if $(i, i+m+3) \in$ $\vec{C}_{3 m}^{3, m+3}$, then $(f(i), f(i+m+3)) \in \vec{C}_{3 m}^{3,2 m+3}$. Since $f(i)$ is a one-one function from $[0,3 m-1]$ into itself we have exhibited an isomorphism. 
Note that if $3 \mid m$, then $T\left(\vec{C}_{3 m}^{3,2 m+3}\right)=0$. We have thus proved $T\left(\vec{C}_{3 m}^{3, m+3}\right)=T\left(\vec{C}_{3 m}^{3,2 m+3}\right)= \begin{cases}m\left(2^{3 m-1}+2^{m-1}-2^{2 m} \cos \frac{m}{3} \pi\right) & \text { if } 3 \nmid m, \\ 0 & \text { otherwise }\end{cases}$

Derivations of (i), (ii), (iii), (iv), (v), (vi)

In (a), (b), and (c) below we derive the formulas for the numbers of spanning trees in the above six graphs to verify the validity of our claim.

(a) $T\left(\vec{C}_{3 m}^{2,2 m+2}\right)$ and $T\left(\vec{C}_{3 m}^{1, m+1}\right) \cdot((\mathrm{i})$ and $(\mathrm{v}))$

We already saw that when $m$ is even $T\left(\vec{C}_{3 m}^{2,2 m+2}\right)=0$. We also saw that when $m$ is odd $\vec{C}_{3 m}^{2,2 m+2}$ is isomorphic to $\vec{C}_{3 m}^{1, m+1}$. We therefore only have to evaluate the number of spanning trees for $\vec{C}_{3 m}^{1, m+1}$.

In this case $p=1, d_{1}=3, d_{2}=1, \beta=1$ so $v=m, d_{1}^{\prime}=d_{1} / \beta=3$. We only need to derive the two functions $\delta_{\ell v}=X_{\ell}(v), \ell=1,2$. Now $d_{2}^{\prime}=d_{2} / \beta=1, \bar{d}_{2}^{\prime}=2$. We therefore find $x_{1}=1, \quad x_{2}=2$. Substituting into (10) yields

$$
\begin{aligned}
& Y_{1}(v)=v\left(2^{v}+2 \cos \frac{v-2}{3} \pi\right), \\
& Y_{2}(v)=v\left(2^{2 v}+2(-1)^{v} \cos \frac{v-2}{3} \pi\right) .
\end{aligned}
$$

The system of equations (8) in this case is

$$
\begin{aligned}
-Y_{1}(v) & =(-1)^{v} v X_{1}(v) \\
-Y_{2}(v) & =(-1)^{v} X_{1}(v) Y_{1}(v)+(-1)^{2 v} 2 v X_{2}(v)
\end{aligned}
$$

and solving for $X_{\ell}(v)$ yields

$$
\begin{aligned}
& X_{1}(v)=-(-1)^{v}\left(2^{v}+2 \cos \frac{v-2}{3} \pi\right), \\
& X_{2}(v)=\left(2^{v+1}-(-1)^{v}\right) \cos \frac{v-2}{3} \pi+2 \cos ^{2} \frac{v-2}{3} \pi .
\end{aligned}
$$

Equation (11) states

$$
T\left(\vec{C}_{3 m}^{1, m+1}\right)=3 m 2^{3 m-1}+(-1)^{m} 2 m \delta_{m} 2^{2 m-1}+(-1)^{2 m} m \delta_{2 m} 2^{m-1} .
$$


Substituting in the values $\delta_{\ell m}=X_{\ell}(m)$ and simplifying yields

$$
T\left(\vec{C}_{3 m}^{1, m+1}\right)=m\left(2^{3 m-1}+2^{m-1}-2^{2 m} \cos \frac{m-2}{3} \pi\right)
$$

proving (i) and (v).

(b ) $T\left(\vec{C}_{3 m}^{1,2 m+1}\right)$ and $T\left(\vec{C}_{3 m}^{2, m+2}\right)(($ ii) and (iv))

We already saw that when $m$ is even $T\left(\vec{C}_{3 m}^{2, m+2}\right)=0$ and, when $m$ is odd, $\vec{C}_{3 m}^{2, m+2}$ is isomorphic to $\vec{C}_{3 m}^{1,2 m+1}$. We therefore only have to calculate the number of spanning trees in $\vec{C}_{3 m}^{1,2 m+1}$. In this case $p=1, d_{1}=3$, $d_{2}=2, \beta=1$ so $v=m, d_{1}^{\prime}=d_{1} / \beta=3$. We therefore only need to derive the two functions $\delta_{\ell v}=X_{\ell}(v), \ell=1,2$. Now $d_{2}^{\prime}=d_{2} / \beta=2, \bar{d}_{2}^{\prime}=d_{2}^{\prime}=2$. We find $x_{1}=2, \quad x_{2}=1$. Substituting into (10) yields

$$
\begin{aligned}
& Y_{1}(v)=v\left(2^{v}+2 \cos \frac{v-4}{3} \pi\right), \\
& Y_{2}(v)=v\left(2^{2 v}+2(-1)^{v} \cos \frac{v-4}{3} \pi\right) .
\end{aligned}
$$

The system of equations (8) in this case is again

$$
\begin{aligned}
& -Y_{1}(v)=(-1)^{v} v X_{1}(v) \\
& -Y_{2}(v)=(-1)^{v} X_{1}(v) Y_{1}(v)+(-1)^{2 v} 2 v X_{2}(v)
\end{aligned}
$$

and solving for $X_{\ell}(v)$ yields

$$
\begin{aligned}
& X_{1}(v)=-(-1)^{v}\left(2^{v}+2 \cos \frac{v-4}{3} \pi\right), \\
& X_{2}(v)=\left(2^{v+1}-(-1)^{v}\right) \cos \frac{v-4}{3} \pi+2 \cos ^{2} \frac{v-4}{3} \pi .
\end{aligned}
$$

Equation (11) states

$$
T\left(\vec{C}_{3 m}^{1,2 m+1}\right)=3 m 2^{3 m-1}+(-1)^{m} 2 m \delta_{m} 2^{2 m-1}+(-1)^{2 m} m \delta_{2 m} 2^{m-1} .
$$

Substituting in the values $\delta_{\ell m}=X_{\ell}(m)$ and simplifying yields

$$
\begin{aligned}
T\left(\vec{C}_{3 m}^{1,2 m+1}\right) & =m\left(2^{3 m-1}+2^{m-1}-2^{2 m} \cos \frac{m-4}{3} \pi\right) \\
& =m\left(2^{3 m-1}+2^{m-1}+2^{2 m} \cos \frac{m-1}{3} \pi\right)
\end{aligned}
$$


where the last equality comes from the fact $\cos (x-\pi)=-\cos x$. This proves (ii) and (iv).

$$
\text { (c ) } T\left(\vec{C}_{3 m}^{3,2 m+3}\right) \text { and } T\left(\vec{C}_{3 m}^{3, m+3}\right)((\text { iii) and (vi)) }
$$

We already saw that when $3 \mid m$ both graphs have no spanning trees and when $3 \nmid m$ the two graphs are isomorphic. We therefore only consider $T\left(\vec{C}_{3 m}^{3,2 m+3}\right)$. In this case $p=3, d_{1}=3, d_{2}=2, \beta=1$ so $v=m$, $d_{1}^{\prime}=d_{1} / \beta=3$. We therefore only need to derive the two functions $\delta_{\ell v}=X_{\ell}(v), \ell=1,2$. Now $d_{2}^{\prime}=d_{2} / \beta=2, \bar{d}_{2}^{\prime}=d_{2}^{\prime}=2$. We find $x_{1}=0, \quad x_{2}=0$. Substituting into (10) yields

$$
\begin{aligned}
& Y_{1}(v)=v\left(2^{v}+2 \cos \frac{v}{3} \pi\right), \\
& Y_{2}(v)=v\left(2^{2 v}+2(-1)^{v} \cos \frac{v}{3} \pi\right)
\end{aligned}
$$

The system of equations (8) in this case is yet again

$$
\begin{aligned}
& -Y_{1}(v)=(-1)^{v} v X_{1}(v) \\
& -Y_{2}(v)=(-1)^{v} X_{1}(v) Y_{1}(v)+(-1)^{2 v} 2 v X_{2}(v)
\end{aligned}
$$

and solving for $X_{\ell}(v)$ yields

$$
\begin{aligned}
& X_{1}(v)=-(-1)^{v}\left(2^{v}+2 \cos \frac{v}{3} \pi\right), \\
& X_{2}(v)=\left(2^{v+1}-(-1)^{v}\right) \cos \frac{v}{3} \pi+2 \cos ^{2} \frac{v}{3} \pi .
\end{aligned}
$$

Equation (11) states

$$
T\left(\vec{C}_{3 m}^{3,2 m+3}\right)=3 m 2^{3 m-1}+(-1)^{m} 2 m \delta_{m} 2^{2 m-1}+(-1)^{2 m} m \delta_{2 m} 2^{m-1} .
$$

Substituting in the values $\delta_{\ell m}=X_{\ell}(m)$ and simplifying yields (if $3 \nmid m$ )

$$
T\left(\vec{C}_{3 m}^{3,2 m+3}\right)=m\left(2^{3 m-1}+2^{m-1}-2^{2 m} \cos \frac{m}{3} \pi\right),
$$

proving (iii) and (vi).

Acknowledgments: The authors would like to thank the referees for their careful reading of our original manuscript and for their several suggestions towards improving the presentation of the paper. This work 
was partially supported by Hong Kong CERG grants HKUST6082/97E, HKUST6137/98E, HKUST6162/00E and HKUST DAG98/99.EG23. The first author's work was also supported by DIMACS, Rutgers University; the second author's by The Natural Science Grant of Gansu Province (3SZ051-A25-037).

\section{References}

[1] J. C. Bermond, F. Comellas, and D. F. Hsu, Distributed loop computer networks: A survey, J. Parallel Distrib. Comput. 24 (1995), $2-10$.

[2] N. Biggs, Algebraic graph theory, 2nd Edition, Cambridge University Press, London, 1993.

[3] F. T. Boesch and H. Prodinger, Spanning tree formulas and Chebyshev polynomials, Graph and Combinatorics 2 (1986), 191-200.

[4] D. Cvetkovič, M. Doob, and H. Sachs, Spectra of graphs: Theory and applications, Third Edition, Johann Ambrosius Barth, Heidelberg, 1995.

[5] P. Erdös and D. F. Hsu, Distributed loop networks with minimum transmission delay, Theoretical Computer Science 100 (1992), 223241.

[6] J. Fàbrega and M. Zaragozà, Fault-tolerant routings in double fixedstep networks, Discrete Applied Math. 78 (1997), 61-74.

[7] M. J. Golin, Y. C. Leung, and Y. J. Wang, Counting spanning trees and other structures in non constant-jump circulant graphs, The 15th Annual International Symposium on Algorithms and Computation, 2004, pp. 508-521.

[8] F. Harary, Graph theory, Addison-Wesley, Reading MA, 1969.

[9] F. K. Hwang, A survey on double loop networks, Proceedings DIMACS Workshop on Reliability of Computer and Communication Networks, 1989, pp. 143-151. 
[10] W. Jennings, First course in numerical methods, The Macmillan Co., New York, 1964.

[11] G. Kirchhoff, Über die Auflösung der gleichungen, auf welche man bei der untersuchung der linearen verteilung galvanischer Ströme geführt wird, Ann. Phys. Chem. 72 (1847), 497-508.

[12] M. T. Liu, Distributed loop computer networks, Advanced in Computers 17 (1978), 163-221.

[13] Z. Lonc, K. Parol, and J. M. Wojciechowski, On the number of spanning trees in directed circulant graphs, Networks 37 (2001), 129-133.

[14] R. Vohra and L. Washington, Counting spanning trees in the graphs of Kleitman and Golden and a generalization, J. Franklin Inst. 318 (1984), 349-355.

[15] X. Yong and F. J. Zhang, An asymptotic behavior of the complexity of double fixed step loop networks, Applied Math. J. Chinese Univ. Ser. B 12 (1997), 233-236.

[16] F. J. Zhang and X. Yong, Asymptotic enumeration theorems for the numbers of spanning trees and Eulerian trails in circulant digraphs and graphs, Science in China, Ser. A 43 (1999), 264-271.

[17] Y. P. Zhang, X. Yong, and M. J. Golin, The number of spanning trees in circulant graphs, Discrete Math. 223 (2000), 337-350. 